\title{
Synthesis of Silver Nanoparticles Using a Microfluidic Impinging Jet Reactor
}

\author{
Razwan Baber ${ }^{1}$, Luca Mazzei ${ }^{1}$, Nguyen T.K. Thanh ${ }^{2,3}$ and Asterios Gavriilidis ${ }^{1 *}$ \\ ${ }^{1}$ Department of Chemical Engineering, University College London, Torrington Place, London WC1E 7JE, UK \\ ${ }^{2} U C L$ Healthcare Biomagnetic and Nanomaterials Laboratories, 21 Albemarle Street, London W1S 4BS, UK \\ ${ }^{3}$ Biophysics Group, Department of Physics and Astronomy, University College London, Gower Street, London WC1E 6BT, UK
}

Received: 28 April 2016; accepted: 16 June 2016

\begin{abstract}
Synthesis of silver nanoparticles (NPs) in an impinging jet reactor (IJR) was investigated due to its unique properties of efficient mixing and lack of channel walls which avoid fouling. Silver NPs were formed at room temperature by reducing silver nitrate with sodium borohydride in the presence of sodium hydroxide. Two types of ligand were used to stabilize the NPs, trisodium citrate, and polyvinyl alcohol (PVA). Weber number, the ratio between inertial forces and surface tension forces, is used to characterize flow in impinging jets. Flow regimes were investigated for Weber numbers in the range of 13176. A liquid sheet/chain regime was identified at lower Weber numbers $(<90)$, and an unstable rim structure was identified at higher Weber numbers ( $>90)$. Mixing time was found to be in the range 1-7 ms, using the Villermaux-Dushman reaction system and interaction by exchange with the mean mixing (IEM) model. Fastest mixing occurred at Weber number ca. 90. Using trisodium citrate as a ligand, NP size decreased from $7.9 \pm 5.8 \mathrm{~nm}$ to $3.4 \pm 1.4 \mathrm{~nm}$ when flow rate was increased from $32 \mathrm{~mL} / \mathrm{min}$ to $72 \mathrm{~mL} / \mathrm{min}$ using $0.5 \mathrm{~mm}$ jets, and from $6.4 \pm 3.4 \mathrm{~nm}$ to $5.1 \pm 4.6 \mathrm{~nm}$ when flow rate was increased from $20 \mathrm{~mL} / \mathrm{min}$ to $32 \mathrm{~mL} / \mathrm{min}$ using $0.25 \mathrm{~mm}$ jets. Using PVA as a ligand, NP size decreased from $5.4 \pm 1.6 \mathrm{~nm}$ to $4.2 \pm 1.1 \mathrm{~nm}$ using $0.5 \mathrm{~mm}$ jets and stayed relatively constant between $4.3 \pm 1 \mathrm{~nm}$ and $4.7 \pm 1.3 \mathrm{~nm}$ using $0.25 \mathrm{~mm}$ jets. In general, the size of the NPs decreased when mixing was faster.
\end{abstract}

Keywords: mixing time, impinging jets, nanomaterials, nanocrystallization

\section{Introduction}

Nanoparticles (NPs) display unique properties due to their high surface area to volume ratios and quantum effects. Because NPs have a large percentage of their atoms present on their surface, they offer very different properties to their corresponding bulk materials allowing for a multitude of potential applications [1]. Silver NPs find applications as antimicrobial agents [2], in catalysis [3], surface-enhanced Raman scattering [4], electronics [5], and sensors [6]. The study of how the size, shape, and dispersity of the NPs can be manipulated is important since the properties the particles display depend on these parameters. These morphological properties of the NPs are largely controlled by a chemical reaction and subsequent nucleation and growth [7]. These are in turn controlled by parameters such as concentration of reagents, $\mathrm{pH}$, and temperature. Usually, a change in morphological parameters is brought about by changing one of the above parameters. To obtain a fine control over the morphology of the NPs, the processes of mass and heat transfer must be carefully controlled. Traditionally, batch reactors have been used to explore new synthetic routes to obtain a variety of different NP shapes such as spheres [8], plates [9], disks [10], rods [11], flowers [12], urchins [13], octopods [14], wires [15], and core-shell structures [16]. Besides the shape and composition of the NPs, the dispersity is also important, where highly monodisperse particles are usually desirable. Flow reactors offer some distinct advantages over batch reactors for the manipulation of morphology of NPs such as spatiotemporal separation, meaning that stages of reduction reaction, nucleation, and growth can be separated along the length of the reactor [17]. This property allows for a more precise manipulation of the processes to enable tighter control over NP characteristics. An advantage of microfluidic devices in particular is that they offer improved heat and mass transfer because of their smaller characteristic lengths [18]. There are excellent review papers that offer various examples of nanomaterials synthesis using a variety of microfluidic devices [19].

The synthesis of silver NPs using sodium borohydride as a reducing agent typically leads to small NPs because borohydride

\footnotetext{
* Author for correspondence: a.gavriilidis@ucl.ac.uk
}

is a strong reducing agent; however, it tends to give polydisperse NPs. Our previous study using a coaxial flow reactor produced silver NPs in the 4-10 $\mathrm{nm}$ range and showed that mixing is an important parameter when using borohydride as a reducing agent [20]. Split and recombine type flow reactors [21] and polytetrafluoroethylene (PTFE) flow chambers [22] have been utilized to synthesize silver NPs using borohydride obtaining NPs in the range of 10-20 $\mathrm{nm}$ for borohydride to silver nitrate ratios ranging from 3 to 40. Batch reactor syntheses of silver NPs using borohydride as a reducing agent produced NPs in the $1-100 \mathrm{~nm}$ range $[8,23]$.

A non-confined impinging jet reactor (IJR) with characteristic dimensions in the micrometer range is employed in this study to investigate how manipulating mass transfer affects the NP characteristics, in particular, the size and dispersity. IJR is a particularly useful device to use in the synthesis of NPs since it does not clog because of absence of channel walls. Fouling is particularly problematic in microfluidic devices since their small dimensions make them potentially vulnerable to blockage, and various strategies in the literature have been used to avoid fouling such as segmented flow [24], coaxial flow [20, 25], and surface silanization [26].

The IJR has found most common application in bipropellant liquid rocket engines [27], but it also presents some very distinct advantages for reactions which produce solids. Mahajan and Kirwan characterized the mixing times in an IJR using the Bourne reaction scheme [28]. They subsequently carried out a precipitation reaction for the synthesis of lovastatin crystals and compared the size of crystals obtained when the mixing time was (1) slower and (2) faster than the induction time for crystallization. They concluded that a more monodisperse particle size distribution (PSD) was obtained when mixing time was faster. They also concluded that, if the mixing time is faster than the induction time (elapsed time period between achievement of supersaturation and the appearance of crystals) [29], further improvements to the mixing time do not significantly affect the resultant crystals. Erni and Elabbadi studied the hydrodynamic properties of the IJR and characterized various flow regimes before using the IJR for an acid-induced crystallization of sodium benzoate. They found a decrease in both size and dispersity with an increasing jet velocity 
[30]. Kumar et al. employed an IJR for the synthesis of nanocrystalline $\mathrm{MgO}$, a process which yields a rigid gel which can quickly clog microchannels. A change in impingement angle and velocity of the jets was found to affect the surface area of the nanocrystalline $\mathrm{MgO}$ formed, and for each parameter, there was an optimum that existed to obtain maximum surface area. It was suggested that this optimum existed because of a change in hydrodynamics in the impingement zone, where too high a flow rate or impingement angle led to non-optimal mixing conditions, resulting in a reduced contact time for reaction [31]. Hosni et al. used impinging jet reactors for the synthesis of ZnO NPs, comparing results against those formed in a batch stirred vessel and a T-mixer type reactor. The size of the NPs synthesized in each reactor depended on the energy dissipation used for mixing, with the IJR having intermediate energy dissipation versus the other two types of reactors and, hence, producing an intermediate size of $\mathrm{ZnO}$ NPs [32].

Confined impinging jet reactors (CIJRs) are a similar type of reactor for use in reactions involving precipitation of solids. Johnson and Prud'homme employed a CIJR for the precipitation of hydrophobic organic actives NPs; importantly the CIJR allows for homogeneous conditions prior to precipitation of NPs (i.e., formation of block copolymer NPs and nucleation and growth of organic actives) which ultimately separates the effect of mixing on the final size of the NPs [33]. The NP size decreased with increasing mixing efficiency up until a "breakpoint" was reached, at which point the size of the block copolymer NPs remained constant. They also observed the same effect when using a different block copolymer, and the "breakpoint" corresponded to a Dahmköhler number of 1 [34]. Marchisio et al. studied the precipitation of barium sulfate NPs in a CIJR and similarly observed a reduction in particle size with increasing mixing intensity, but no further improvements were observed when the characteristic mixing time scale reached the reaction time scale [35]. Reduction in size of precipitated barium sulfate NPs with increasing mixing intensity was also observed by Schwarzer and Peukert using a Tmixer [36]. Siddiqui et al. used a CIJR for the synthesis of iron oxide NPs where they found a reduction from $800 \mathrm{~nm}$ to $200 \mathrm{~nm}$ in agglomerate size by increasing the flow rate under high concentration conditions [37]. These studies show the importance of mixing for reactions which lead to formation of NPs, since they typically have reaction times that are small and, hence, need very good mixing efficiency to approach monodispersity. According to the literature above, a reduction in NP size is reported with increasing mixing efficiency attributed to increasing supersaturation levels which would favor a higher nucleation rate and, hence, smaller particles.

The IJR used in this study presents a convenient platform for the synthesis of silver NPs because the reactor has no channel walls in the reaction zone, eliminating the need for lengthy cleaning procedures which are needed in traditional microchannels because of fouling. The IJR can synthesize silver NPs in a highly repeatable manner, associated with the lack of fouling which can occur in other types of reactors where surfaces are present near the reaction zone. The main objective of this work is to investigate the effect of mixing on the size and dispersity of silver NPs synthesized using two different ligands: (1) trisodium citrate and (2) polyvinyl alcohol (PVA). The hydrodynamics and mixing efficiency of the IJR have also been studied.

\section{Methodology}

2.1. Chemicals. Silver nitrate $\left(\mathrm{AgNO}_{3}, 0.01 \mathrm{M}\right.$ stock solution), trisodium citrate $\left(\mathrm{HOC}(\mathrm{COONa})\left(\mathrm{CH}_{2} \mathrm{COONa}\right)_{2} \cdot 2 \mathrm{H}_{2} \mathrm{O}\right.$, powder form, PVA (molecular weight 89,000-98,000 Da), sodium borohydride solution $\mathrm{NaBH}_{4}, \sim 12$ wt.\% in $14 \mathrm{M} \mathrm{NaOH}$ stock solution), potassium iodide (KI, 99\%), potassium iodate $\left(\mathrm{KIO}_{3}, 98 \%\right.$ ), boric acid $\left(\mathrm{H}_{3} \mathrm{BO}_{3}, 99.5 \%\right)$, and sulfuric acid $\left(\mathrm{H}_{2} \mathrm{SO}_{4}, 3 \mathrm{M}\right.$ stock solution) were obtained from Sigma. All chemicals were used without further purification, and solutions were prepared with ultrapure water (resistivity, $15.0 \mathrm{M} \Omega \mathrm{cm}$ ).

2.2. Experimental Setup. Syringe pumps (Pump 11 Elite OEM module, Harvard) were used in the flow experiments to deliver the reagents to the IJR. Figure 1 shows a schematic representation of the IJR, which consisted of two stainless steel tubes with equal internal diameters of $0.25 \mathrm{~mm}$ or $0.5 \mathrm{~mm}$ aligned at an angle, $\theta=48^{\circ}$. The outer diameters of the tubing were $1.59 \mathrm{~mm}$. Spacing between the centers of the fluid jets at the point of tube outlets, $d$, was $3 \mathrm{~mm}$.

2.3. Nanoparticle Synthesis. A mixture was made by combining silver nitrate and ligand (trisodium citrate or PVA) into a solution and diluting to the appropriate concentration for input 1 (Figure 1) and diluting sodium borohydride/sodium hydroxide solution to the appropriate concentration for input 2 . Since the sodium borohydride was stored in $14 \mathrm{M}$ sodium hydroxide, the concentration of sodium hydroxide was 3.21 times higher than the stated sodium borohydride concentration in all cases. The syringe pumps delivered reagents to input 1 and input 2 at the appropriate flow rates to produce the desired jets. As the two jets collide, the reagents mix followed by subsequent reaction and formation of silver NPs. Reactions were carried out at room temperature $\left(22-24^{\circ} \mathrm{C}\right)$. All concentrations stated are those at the inlets before any mixing of reagents occurs.

2.4. Characterization of Nanoparticles. The silver NPs were analyzed using an ultraviolet-visible (UV-vis) spectrometer (USB 2000+ Spectrometer and DT-Mini-2-GS light source, Ocean Optics) between 45 and 60 min after synthesis (the signal of the samples were stable in this window of time). Silver NP samples were diluted with additional ultrapure water to bring the absorbance into a suitable range (i.e., obeying the BeerLambert law and avoiding saturation of the light detector), and the data were normalized so that the maximum absorbance in the flow rate range tested represented an absorbance of 1 . Transmission electron microscope images were captured using a JEOL 1200 EX ii microscope with a $120-\mathrm{kV}$ acceleration voltage. Carbon coated copper transmission electron microscope (TEM) grids were prepared between 45 and 60 min after synthesis by pipetting a $5-\mu \mathrm{L}$ sample onto the grid and allowing it to dry at room temperature. Particle size distributions (insets for each TEM image presented) have the following nomenclature: $d$ is average diameter, $\delta d$ is the standard deviation of the NP distribution, and $n$ is the number of particles counted to obtain the particle size distribution.

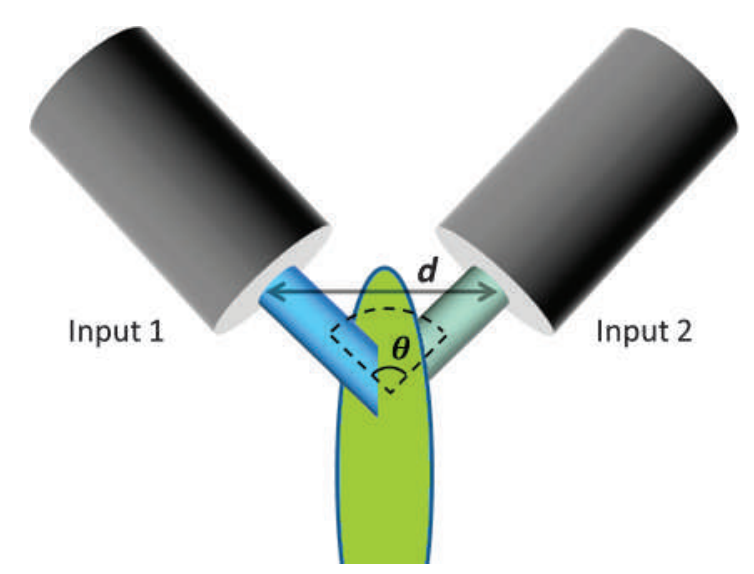

Figure 1. Representation of the impinging jet reactor 


\section{Results and Discussion}

3.1. Flow and Mixing Characterization. The VillermauxDushman test reaction system is a well-known method to characterize mixing efficiency in a reactor [38]. Mixing time within a micromixing device can be obtained using this reaction system by tuning the concentrations appropriately. The Villermaux-Dushman reaction system can be described as follows. A stoichiometric defect of sulfuric acid is introduced to a mixture of borate, iodide, and iodate ions. The following reactions occur:

$$
\begin{aligned}
& \mathrm{H}_{2} \mathrm{BO}_{3}^{-}+\mathrm{H}^{+} \leftrightarrow \mathrm{H}_{3} \mathrm{BO}_{3} \\
& 5 \mathrm{I}^{-}+\mathrm{IO}_{3}^{-}+6 \mathrm{H}^{+} \leftrightarrow 3 \mathrm{I}_{2}+3 \mathrm{H}_{2} \mathrm{O} \\
& \mathrm{I}_{2}+\mathrm{I}^{-} \leftrightarrow \mathrm{I}_{3}^{-}
\end{aligned}
$$

instantaneous very fast

Reaction 1 is much faster than reaction 2 , and since there is a stoichiometric defect of acid, only reaction 1 occurs if there is perfectly efficient mixing. In practice, reaction 2 increases as the mixing efficiency decreases because iodide and iodate will react with acid if the local concentration of orthoborate ions has been depleted (this only occurs if the mixing is not perfectly efficient). Reaction 2 produces iodine, which further reacts with iodide ions to form triiodide (reaction 3). A defect of acid amount is needed because the mean $\mathrm{pH}$ must be within a narrow range close to the value for the iodine dismutation $\mathrm{pH}\left(\mathrm{pH}^{*}\right)$. If the $\mathrm{pH}$ is lower than $\mathrm{pH}^{*}$, iodine can form in acid solution irrespective of micromixing effects, while, on the other hand, the $\mathrm{pH}$ cannot be too basic since formation of iodine becomes thermodynamically unstable [38]. By measuring the triiodide concentration, the extent to which reaction 2 occurs can be measured, hence allowing the mixing efficiency to be estimated. Triiodide exhibits a strong peak at $353 \mathrm{~nm}$, enabling its concentration to be calculated. The extinction coefficient of triiodide was found to be $23,209 / \mathrm{M} / \mathrm{cm}$ at $353 \mathrm{~nm}$, which is similar to the values reported in the literature (details on this procedure can be found in Supporting Information, and calibration curves for iodine and triiodide are shown in Figure S1 and Figure S2, respectively) [38]. The micromixing quality is quantified by the segregation index which is discussed in detail in other studies [38, 39]. The segregation index can be linked to the mixing efficiency through the use of a phenomenological mixing model. The interaction by exchange with the mean (IEM) model was used to estimate the mixing time from the experimental results (Supporting Information provides more details of the model and curves of segregation index vs. mixing time for both IJRs in Figure S3) [40].

The Villermaux-Dushman reaction system was carried out in the $0.5 \mathrm{~mm}$ and $0.25 \mathrm{~mm}$ IJRs. For input $1,0.017 \mathrm{M}$ and $0.02 \mathrm{M}$ of sulfuric acid were used (corresponding to $0.034 \mathrm{M}$ and $0.04 \mathrm{M} \mathrm{H}^{+}$) for the $0.5 \mathrm{~mm}$ and $0.25 \mathrm{~mm}$ IJR, respectively. The dissociation of sulfuric acid is considered to be almost complete for $\mathrm{pH}$ above 4 , whereas, if the reactions take place at a lower $\mathrm{pH}$, then the assumption of complete acid dissociation would result in overestimating the mixing efficiency. Commenge and Falk noted that the $\mathrm{pH}$ of the solution (acid and buffer combined) will increase as mixing takes place resulting in more dissociation, while suggesting that, if the reaction takes place at $\mathrm{pH} 2.5$, the mixing time will be underestimated by $25 \%$ [39]. Input 2, which is considered the buffer solution, had a concentration of $0.032 \mathrm{M}$ potassium iodide, $0.006 \mathrm{M}$ potassium iodate, $0.045 \mathrm{M}$ sodium hydroxide, and $0.09 \mathrm{M}$ orthoboric acid. Figure 2 shows mixing time vs. Weber number obtained for the $0.5 \mathrm{~mm}$ and $0.25 \mathrm{~mm}$ IJRs. The Weber number is defined as [41]:

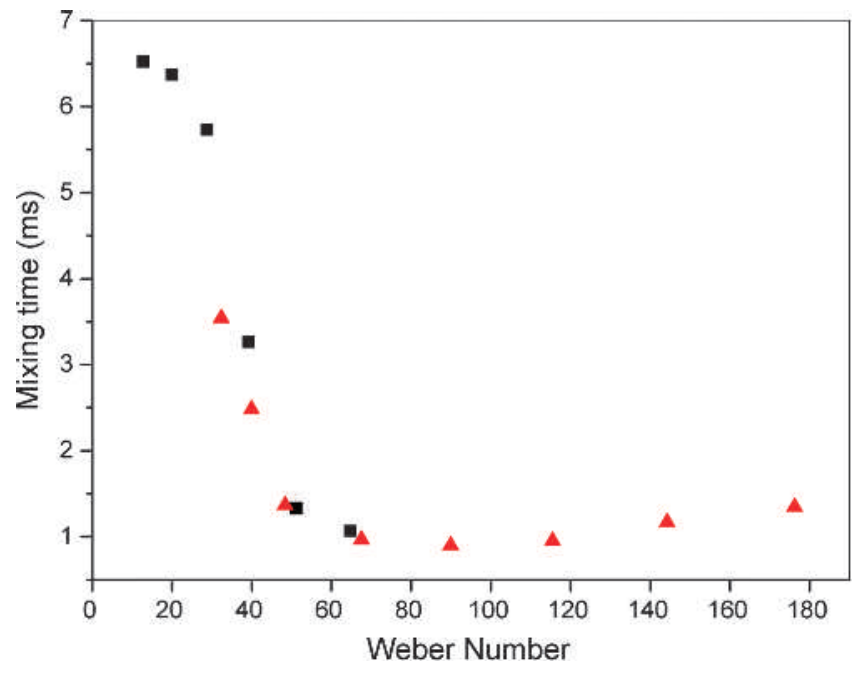

Figure 2. Mixing time calculated by the Villermaux-Dushman reaction scheme vs. Weber number for $0.5 \mathrm{~mm}$ (black squares) and $0.25 \mathrm{~mm}$ (red triangles) IJRs. Concentration of acid solution (input 1) was $0.017 \mathrm{M}$ and $0.02 \mathrm{M} \mathrm{H}_{2} \mathrm{SO}_{4}$ for $0.5 \mathrm{~mm}$ and $0.25 \mathrm{~mm}$ I.D. tubing IJR, respectively. Concentrations of buffer solution (input 2) were $\mathrm{KI}$ : $0.032 \mathrm{M}, \mathrm{KIO}_{3}$ : $0.006 \mathrm{M}, \mathrm{NaOH}$ : $0.045 \mathrm{M}$, and $\mathrm{H}_{3} \mathrm{BO}_{3}: 0.09 \mathrm{M}$ in both IJRs.

$$
\mathrm{We}=\frac{\rho u^{2} d}{\sigma}
$$

where $\rho$ is the density of fluid, $u$ is average velocity of fluid before impact, $d$ is the diameter of the jet (taken as the tube internal diameter), and $\sigma$ is the surface tension of the fluid. Values of fluid properties used are those for water at room temperature. The Weber number represents the ratio of inertial to surface tension forces and is useful in describing what regime the IJR is operating in. The Weber number range for the $0.5 \mathrm{~mm}$ and $0.25 \mathrm{~mm}$ IJRs was $13-65$ and $32-176$, respectively. The Reynolds number is defined as:

$$
\operatorname{Re}=\frac{\rho u d}{\mu}
$$

where $\mu$ is the dynamic viscosity. The Reynolds number represents the ratio of inertial to viscous forces and describes whether the flow is a laminar, intermediate, or turbulent regime. The Reynolds number range for the $0.5 \mathrm{~mm}$ and $0.25 \mathrm{~mm}$ IJRs was 680-1520 and 760-1770, respectively. From Figure 2, it can be seen that the mixing time for both reactors is similar when operating under similar Weber numbers. This indicates that the Weber number is a key parameter in determining the mixing efficiency of an IJR. Erni and Elabbadi propose that the mixing time for an impinging jet is proportional to the Weber number according to a power law relationship $\left(t_{\mathrm{m}} \propto \mathrm{We}^{-0.75}\right)$ [30]. Fitting the data in our study for We $<80$, the following relationship was found, $t_{\mathrm{m}} \propto \mathrm{We}^{-0.89}$. Mixing time decreases with increasing Weber number up to a value of ca. 90 where it reaches a minimum value of around $1 \mathrm{~ms}$. Increasing the Weber number past ca. 90 using the $0.25 \mathrm{~mm}$ IJR results in a gradual increase in mixing time. In general, the specific power dissipation within a reactor can be used to estimate the mixing time, and several studies have shown a correlation between the two $[39,40,42]$. The specific power dissipation for the IJR can be estimated using the following relationships [28]:

$$
\begin{gathered}
\epsilon=\frac{P}{\rho V} \\
P=\frac{1}{2}\left(m_{1} u_{1}^{2}+m_{2} u_{2}^{2}\right)
\end{gathered}
$$




$$
V=\frac{\pi d^{2}}{4} h
$$

where $\epsilon$ is overall energy dissipation per unit mass (W/kg), $P$ is rate of overall mechanical energy dissipation (W) assumed to be equal to the kinetic energies of the two impinging jets, $\rho$ is the fluid density $\left(\mathrm{kg} / \mathrm{m}^{3}\right), V$ is the volume of the impingement zone $\left(\mathrm{m}^{3}\right), m$ is the mass flow rate of a jet $(\mathrm{kg} / \mathrm{s}), u$ is the velocity of a jet $(\mathrm{m} / \mathrm{s})$ (with subscripts 1 and 2 denoting input 1 or 2), $d$ is the diameter (m), and $h$ is the effective thickness of the cylindrical micromixing region. It is difficult to estimate what the cylindrical micromixing region would be, given the non-uniform shape of the liquid sheet after impingement and its changing dimensions depending on the flow rate. If $d$ is assumed to be the jet diameter of the IJR while the value of $h$ to be $10 \%$ of the internozzle distance [28], mixing time can be estimated using the correlation suggested by Commenge and Falk [39]:

$$
t_{\mathrm{m}}=0.15 \epsilon^{-0.45}
$$

where $t_{\mathrm{m}}$ is the mixing time (s). Using the correlation, the mixing time estimate was found to be between $0.9-2.6 \mathrm{~ms}$ for the $0.5 \mathrm{~mm}$ IJR and $0.25-0.9 \mathrm{~ms}$ for the $0.25 \mathrm{~mm}$ IJR for the conditions used in this study. The mixing times found using the IEM model were between $1-6.5 \mathrm{~ms}$ for the $0.5 \mathrm{~mm}$ IJR and $0.9-3.5 \mathrm{~ms}$ for the $0.25 \mathrm{~mm}$ IJR. Considering that both the correlation and the IEM model only give an estimate of the mixing time, the order of magnitude agreement is satisfactory. Increasing specific power dissipation by increasing flow rate within the flow reactor should decrease mixing time. However, this is not the case for the IJR where the mixing time levels off and begins to increase past a We $\sim 90$, most likely because of droplet formation and breakup at the liquid rim of the sheet formed on impingement of the jets. Erni and Elabbadi observed that there is a transition from the liquid sheet to a fragmented sheet (unstable rim) at a We $\sim 30$ at a surfactant concentration of $8.2 \mu \mathrm{M}$ sodium dodecyl sulfate (SDS), We $\sim 80$ at a concentration of $0.82 \mathrm{mM}$, and $\mathrm{We} \sim 200$ at $82 \mathrm{mM}$ [30]. They observed that the mixing quality deteriorates when there is excessive droplet fragmentation; droplets ejected from the sheet have little time for efficient mass transfer which is highest in the liquid sheet (they estimate that droplets took $0.6 \mathrm{~ms}$ to be ejected from the liquid sheet). Using the fourth Bourne reaction scheme, they estimated mixing time in their system to be $4.9 \mathrm{~ms}$, which is of the same order of magnitude as our work. The flow regime in our system was subsequently investigated to ascertain where this transition occurs.

The flow regime of the $0.5 \mathrm{~mm}$ and $0.25 \mathrm{~mm}$ IJR was investigated by capturing images of the IJR during operation using a high speed camera (Photron Fastcam SA1.1 high speed camera). Figure 3 shows the flow regime for the $0.5 \mathrm{~mm}$ IJR system, for flow rates between 32 and $72 \mathrm{~mL} / \mathrm{min}(\mathrm{We}=13$ $65)$. From the images, it can be seen that the flow pattern changes across the flow rate range investigated. At the lowest flow rate, the jets collide and coalesce, forming a small sheet. As the flow rate increases, the sheet increases in length and width. Further increase in the flow rate results in a wavy liquid film surrounding the liquid sheet. This behavior has been observed in other studies where a wavy film occurs, although it is usually accompanied by droplets leaving the stable sheet bounded by a liquid rim. This effect arises from RayleighPlateau instability [43]. It is possible that these waves, observed on the liquid rim and sheet, arise from small oscillations in the flow delivered from the syringe pumps. This wavy nature of the liquid sheet is also transferred into the chain of fluid following the initial sheet formed by the collision of the jets. The liquid following the collision of the jets remains continuous in all cases for the $0.5 \mathrm{~mm}$ IJR, with no droplet breakup in the region visualized. Figure 4 shows the flow regime for the $0.25 \mathrm{~mm}$ IJR system, for flow rates between 18 and $42 \mathrm{~mL} / \mathrm{min}$ (We $=32$ 176). At lower flow rates, a stable sheet and liquid rim are
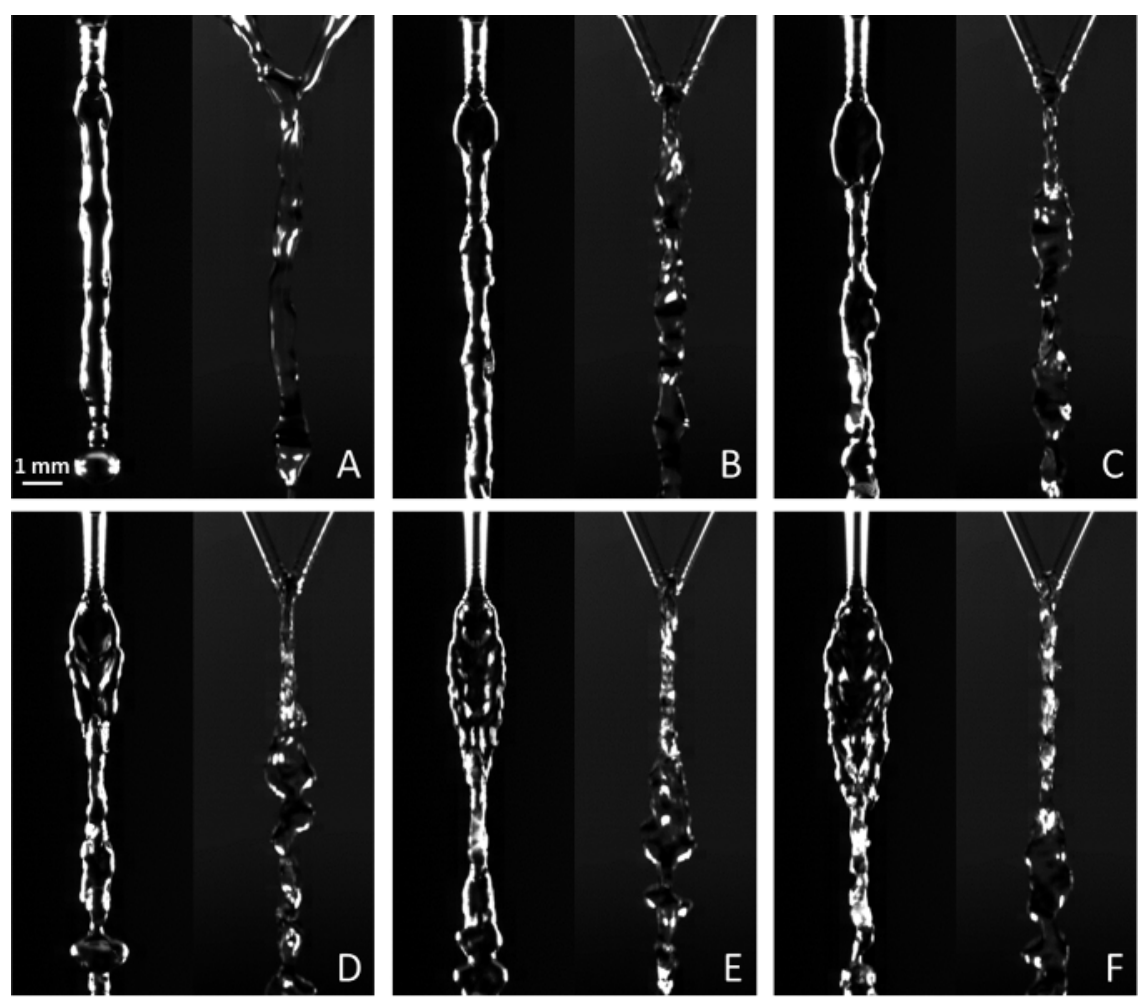

Figure 3. Flow visualization of jets emitted from $0.5 \mathrm{~mm}$ tubes at total flow rate: A: $32 \mathrm{~mL} / \mathrm{min}$ (We: 13$)$, B: $40 \mathrm{~mL} / \mathrm{min}$ (We: 20), C: $48 \mathrm{~mL} / \mathrm{min}$ (We: 29), D: $56 \mathrm{~mL} / \mathrm{min}$ (We: 39), E: $64 \mathrm{~mL} / \mathrm{min}$ (We: 51), and F: $72 \mathrm{~mL} / \mathrm{min}$ (We: 65). Pictures show side view and front view 

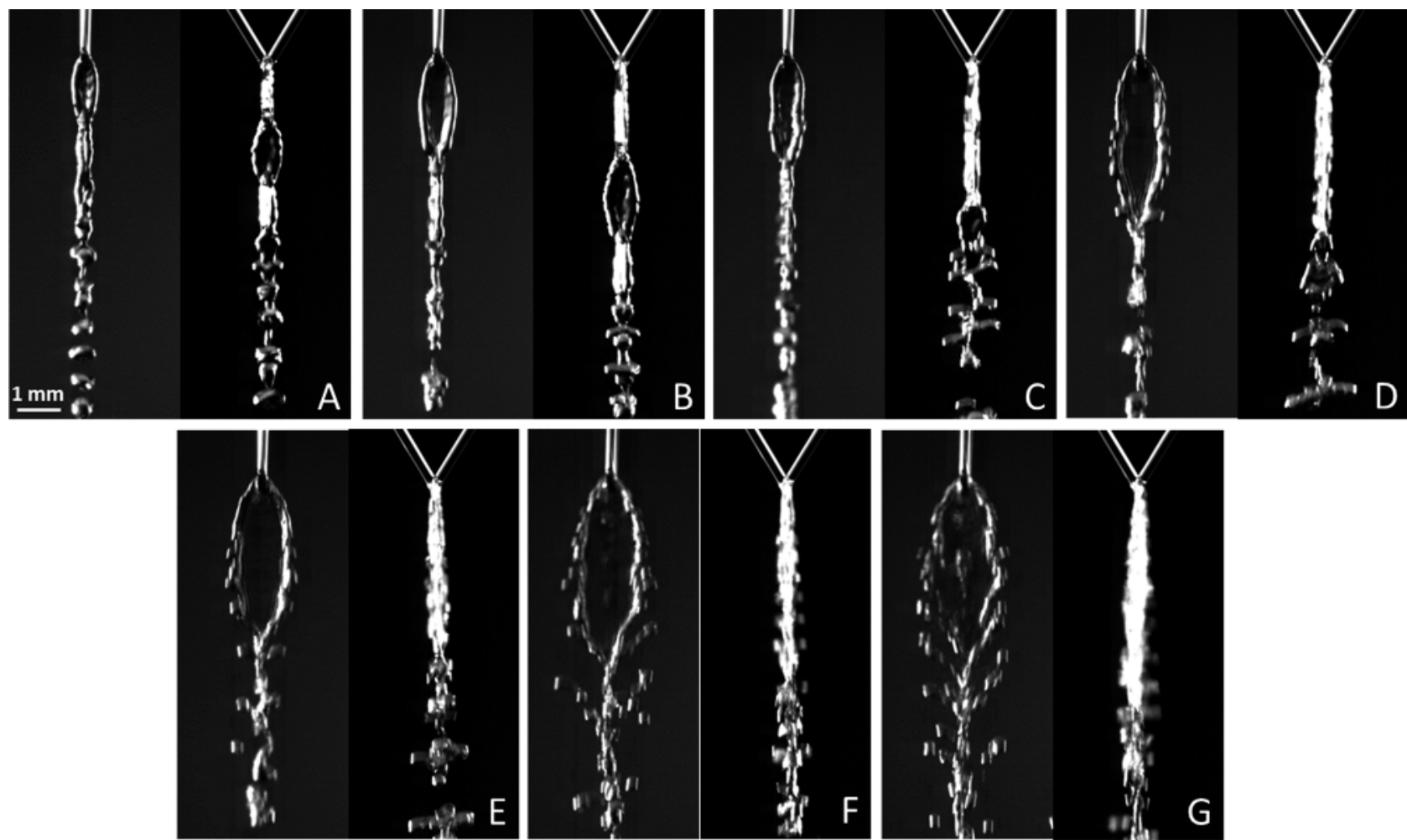

Figure 4. Flow visualization of jets emitted from $0.25 \mathrm{~mm}$ tubes at total flow rate: A: $18 \mathrm{~mL} / \mathrm{min}$ (We: 32 ), B: $22 \mathrm{~mL} / \mathrm{min}$ (We: 48 ), C: $26 \mathrm{~mL} / \mathrm{min}$ (We: 68 ), D: $30 \mathrm{~mL} / \mathrm{min}$ (We: 90), E: $34 \mathrm{~mL} / \mathrm{min}$ (We: 115), F: $38 \mathrm{~mL} / \mathrm{min}$ (We: 144), and G: $42 \mathrm{~mL} / \mathrm{min}$ (We: 176). Pictures show side view and front view

formed at the point of impact. At flow rate above $26 \mathrm{~mL} / \mathrm{min}$, an unstable rim with droplets breaking off the main structure begins to form. From the front view, it can be seen that the flow is also developing a spray in that plane. At this point, the inertial forces overcome the surface tension forces of the fluid resulting in droplet breakaway from the main liquid sheet/rim. Also, for the $0.25 \mathrm{~mm}$ IJR system, the chain of fluid following the liquid sheets after impact is non-continuous after the first few secondary liquid sheets are formed.

Taking the mixing time estimations and flow regime observations into account, the mixing behavior of the IJR system can be rationalized. There are two modes of atomization: reflective and transmitive. Between these two regions there exists a wellmixed atomization condition [44]. In the reflective atomization regime, which occurs at relatively low jet velocities, the jets essentially collide and coalesce forming a thin liquid sheet immediately after collision. Deflection of each jet will be equal as they bounce on each other because of equal momentum. In this region, the turbulence in the mixing layer following impingement increases as flow rate increases leading to an increasing mixing efficiency. However, because of the turbulent nature of the jets, there will be a fluctuation in the trajectory of droplets/sheet as velocity increases; there is an eventual breakup of the liquid sheet where smaller droplets break away. At this point, the atomization transitions into a transmitive mode, whereby droplets are ejected from the liquid rim of the sheet because of increased momentum. This is observed in Figure 4 at We $>90$. The droplets that are ejected from the liquid rim have little contact time in the relatively efficiently mixed sheet, and the mixing efficiency suffers because higher amounts of droplets are ejected. The results show that below a $\mathrm{We} \sim 90$, we are in the reflective atomization regime. Above this Weber number, the regime transitions into a transmitive regime. A We $\sim 90$ seems to yield optimal mixing as confirmed by the mixing time estimation. The observed transition is somewhat higher than those observed by Erni and Elabbadi who, at minimal surfactant concentration, observed transition at We $\sim 30$ [30]. However, they use jets at an angle of $110^{\circ}$ with a jet spacing of $10 \mathrm{~mm}$ which could affect the transition point.

\subsection{Flow Synthesis of Silver NPs}

3.2.1. Effect of Weber Number on Silver NP Size and Dispersity Using Trisodium Citrate as a Stabilizing Agent. The effect that the Weber number has on the silver NPs was investigated in the $0.5 \mathrm{~mm}$ and $0.25 \mathrm{~mm}$ IJRs using trisodium citrate as a stabilizing agent. Concentration of input 1 was $0.9 \mathrm{mM}$ silver nitrate and $6 \mathrm{mM}$ trisodium citrate, and concentration of input 2 was $1.8 \mathrm{mM}$ sodium borohydride. Total flow rates for the $0.5 \mathrm{~mm}$ IJR ranged from $32 \mathrm{~mL} / \mathrm{min}$ to $72 \mathrm{~mL} / \mathrm{min}$, and for the $0.25 \mathrm{~mm} \mathrm{IJR}$, ranged from $18 \mathrm{~mL} / \mathrm{min}$ to $34 \mathrm{~mL} / \mathrm{min}$ (repeatability of the synthesis is shown in Supporting Information, Figure S4).

Figure 5 shows TEM images for NPs synthesized in the $0.5 \mathrm{~mm}$ IJR at $32,48,64$, and $72 \mathrm{~mL} / \mathrm{min}$ where the average diameter and dispersity of the NPs are $7.9 \pm 5.8 \mathrm{~nm}, 7.7 \pm$ $5.0 \mathrm{~nm}, 5.0 \pm 2.8 \mathrm{~nm}$, and $3.4 \pm 1.4 \mathrm{~nm}$. Average diameter and polydispersity decrease with increasing Weber number (flow rate). $t$-Tests showed $p$-values $<0.001$ when comparing all the PSDs against each other. This suggests that the PSDs are all statistically different.

Figure 6 shows TEM images for NPs synthesized in the $0.25 \mathrm{~mm} \mathrm{IJR}$ at 20, 24, 28, and $32 \mathrm{~mL} / \mathrm{min}$ where the average diameter and dispersity of the NPs are $6.4 \pm 3.4 \mathrm{~nm}, 5.9 \pm$ $2.1 \mathrm{~nm}, 5.0 \pm 2.5 \mathrm{~nm}$, and $5.1 \pm 4.6 \mathrm{~nm}$. It appears that, with increasing Weber number (flow rate), the NPs reduce in average size up to ca. $5 \mathrm{~nm}$. $t$-Tests showed $p$-values of $>0.05$ when comparing the PSDs of 20 and $24 \mathrm{~mL} / \mathrm{min}, 24$ and $32 \mathrm{~mL} / \mathrm{min}$, and 28 and $32 \mathrm{~mL} / \mathrm{min}$ (all other $p$-values were $<0.001$ ). These suggest that the PSDs are not statistically different; however, this is most likely down to the large polydispersity. Visual analysis of the TEM images reveals two different populations of NPs, those that are smaller than $20 \mathrm{~nm}$ and those that are larger. Although the larger NPs were present in small frequencies (not larger than 15 counts for any flow rate), there were enough present to affect the average size and dispersity 


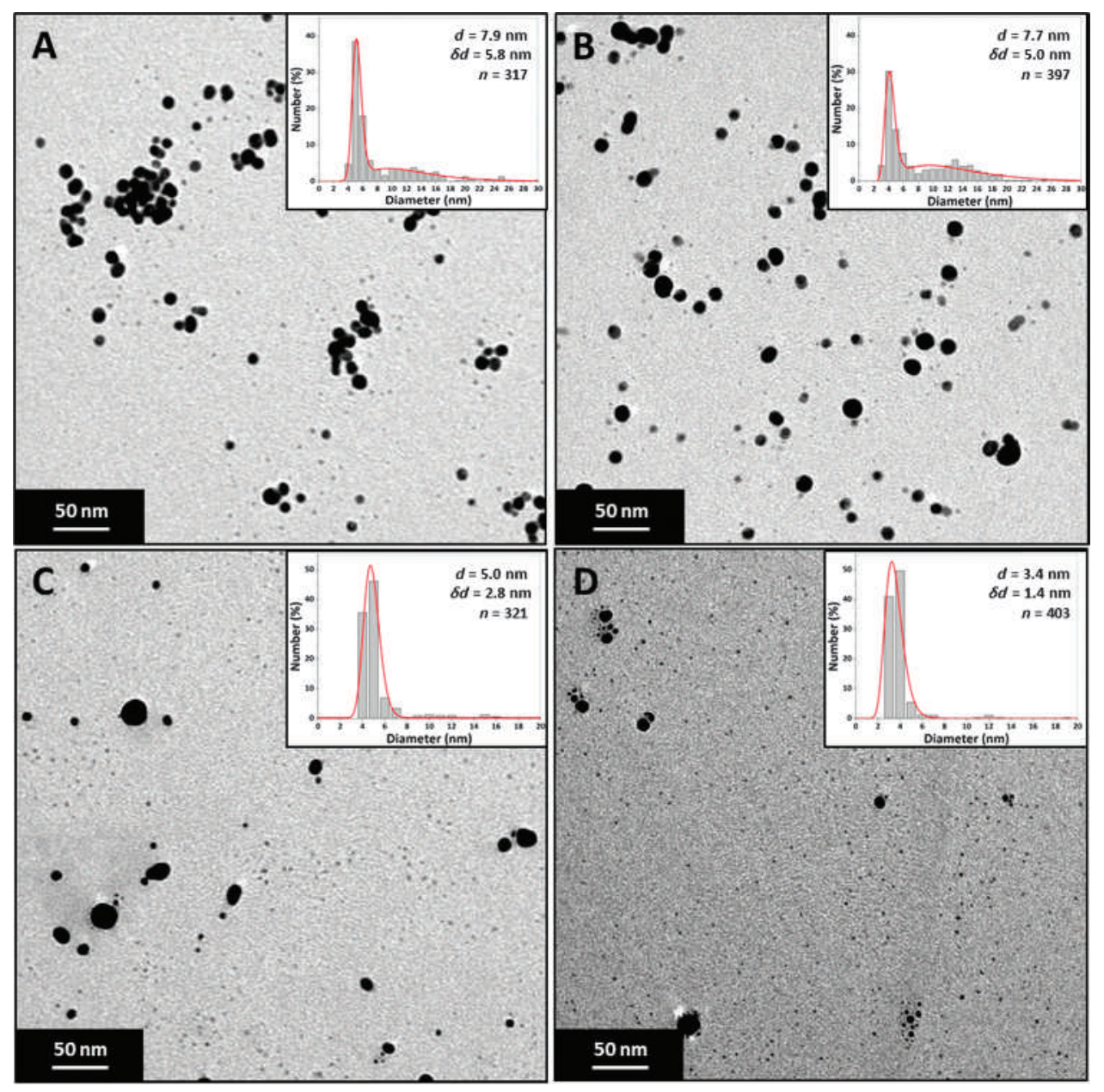

Figure 5. TEM images and particle size distributions of silver NPs synthesized at different total flow rates using a $0.5-\mathrm{mm}$ I.D. tubing IJR. (A) $32 \mathrm{~mL} / \mathrm{min}$ (We: 13), (B) $48 \mathrm{~mL} / \mathrm{min}$ (We: 29), (C) $64 \mathrm{~mL} / \mathrm{min}$ (We: 51), and (D) $72 \mathrm{~mL} / \mathrm{min}$ (We: 65). Concentration of silver nitrate, $0.9 \mathrm{mM}$; trisodium citrate, $6 \mathrm{mM}$ in input 1 ; and sodium borohydride, $1.8 \mathrm{mM}$ in input 2

calculations of NPs synthesized at $20 \mathrm{~mL} / \mathrm{min}$ and $32 \mathrm{~mL} / \mathrm{min}$, but at $24 \mathrm{~mL} / \mathrm{min}$ and $28 \mathrm{~mL} / \mathrm{min}$, virtually no larger NPs are present. If NPs larger than $20 \mathrm{~nm}$ are removed from the $t$-test analysis, only NPs synthesized at $20 \mathrm{~mL} / \mathrm{min}$ and $24 \mathrm{~mL} / \mathrm{min}$ have a $p$-value of $>0.05$ with all other $p$-values being $<0.001$. From the PSDs in Figure 6, it can be seen that, as the Weber number is increased, the average size gets smaller and the dispersion is reduced in the $<20 \mathrm{~nm}$ range. However, the polydispersity is higher at the largest Weber number even though it has the least disperse curve for NPs $<20 \mathrm{~nm}$. This is because synthesis at the highest Weber number produced the highest frequency of larger NPs $(>20 \mathrm{~nm})$, and the disparity in size between the larger and smaller NPs is higher as compared to NPs produced at lower Weber numbers.

Figure 7 shows the peak absorbance vs. Weber number obtained from UV-vis spectroscopy for the silver NPs obtained using the $0.5 \mathrm{~mm} \mathrm{IJR}$ (We $=13-65)$ and the $0.25 \mathrm{~mm} \mathrm{IJR}(\mathrm{We}=$ $32-115)$. It is worth noting that the UV signal did not change significantly $24 \mathrm{~h}$ after synthesis ( $\sim 1 \%$ change in peak absorbance). For each jet diameter, the peak absorbance in general decreases with increasing Weber number. Since the NP size identified from TEM images was primarily below $10 \mathrm{~nm}$, the decreasing peak absorbance is possibly because of a decrease in size of the NPs. For silver NPs, the molar extinction coefficient increases with size [45], and absorbance increases with increasing size below the $10 \mathrm{~nm}$ range, as predicted by Mie theory [46].

The TEM images and peak absorbance of the NPs would suggest that the NPs decrease in size with increasing Weber number while the dispersity shows a more complex relationship. Firstly, the results obtained from the $0.25 \mathrm{~mm}$ IJR will be discussed. In terms of the NP synthesis, the size and dispersity changes can be explained by taking into account mixing characteristics of the impinging jet at the particular Weber number. The NPs below $20 \mathrm{~nm}$ are referred to as smaller NPs and those above $20 \mathrm{~nm}$ are referred to as larger NPs. At low Weber numbers, the smaller NPs tend to be of a larger and more polydisperse nature. As the Weber number increases, the average size of these NPs decreases while the PSDs become tighter. This can be explained through the mixing characteristics within the liquid sheet, where increasing turbulence results in a shorter mixing time. Borohydride has a stabilizing effect on the NPs $[20,46,47]$ and can also supply a high amount of electrons per mole (up to a maximum of eight electrons in theory) [48]. Therefore, if there is efficient mixing within the liquid sheet, there will be a sufficient supply of sodium borohydride in the local regions where reaction is taking place, resulting in increased stabilization and smaller NPs. If there are insufficient amounts of borohydride molecules, less stable NPs, which can grow relatively easily, are formed. Poor local mixing efficiency, which results in low local concentrations of borohydride, will lead to larger NPs, since borohydride can reduce many times its own molar equivalent of silver ions. The formation of larger NPs can be compared to the formation of iodine in the Villermaux-Dushman reactions, which is caused by a local lack of orthoborate ions. Hence, increasing the mixing efficiency (Weber number) decreases the size of the smaller NPs.

It is suggested that the larger NPs are formed in silver nitrate rich regions. The presence of large NPs was most frequent at a 

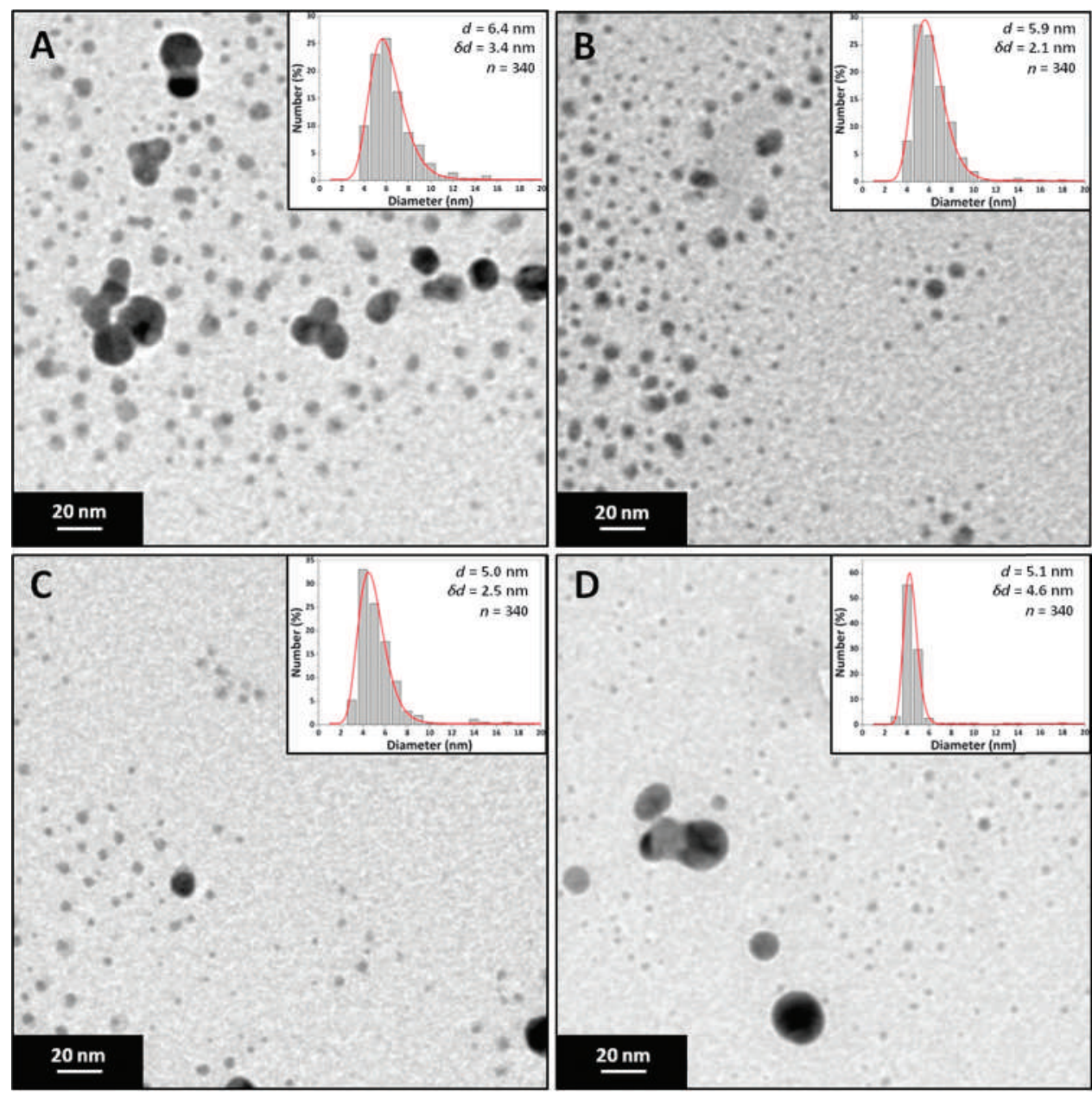

Figure 6. TEM images and particle size distributions of silver NPs synthesized at different total flow rates using a 0.25 -mm I.D. tubing IJR. (A) $20 \mathrm{~mL} / \mathrm{min}$ (We: 40), (B) $24 \mathrm{~mL} / \mathrm{min}$ (We: 58), (C) $28 \mathrm{~mL} / \mathrm{min}$ (We: 78), and (D) $32 \mathrm{~mL} / \mathrm{min}$ (We: 102). Concentration of silver nitrate, $0.9 \mathrm{mM}$; trisodium citrate, $6 \mathrm{mM}$ in input 1 ; and sodium borohydride, $1.8 \mathrm{mM}$ in input 2

Weber number of 40 and 102. Intermediate Weber numbers of 58 and 78 had virtually no NPs over $20 \mathrm{~nm}$. The appearance of larger NPs and their change in size and frequency can be explained by mixing efficiency and the transition from a

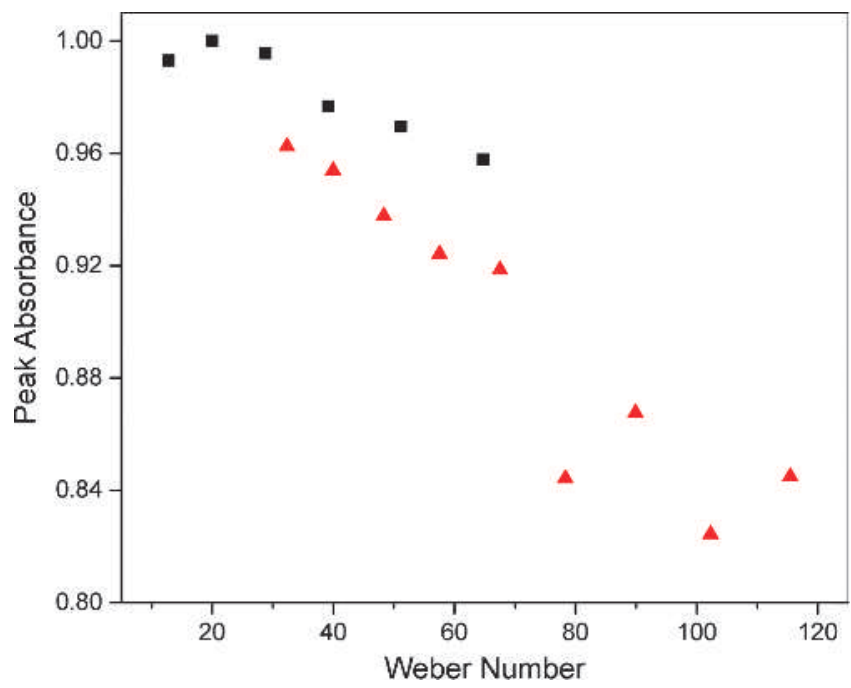

Figure 7. Dependence of peak absorbance (peak wavelength 386$389 \mathrm{~nm}$ ) on Weber number obtained from UV-vis spectroscopy of silver NPs synthesized at different total flow rates using: (1) $0.5 \mathrm{~mm}$ (black squares) and (2) $0.25 \mathrm{~mm}$ I.D. tubing IJR (red triangles). Concentration of silver nitrate, $0.9 \mathrm{mM}$; trisodium citrate, $6 \mathrm{mM}$ in input 1 ; and sodium borohydride, $1.8 \mathrm{mM}$ in input 2 reflective to a transmitive type of atomization. At the lowest Weber numbers, in the reflective atomization regime, there is lower turbulence within the mixing zone. This leads to the possibility of reactions occurring in poorly mixed regions, resulting in the formation of larger NPs. At the intermediate Weber numbers, very few larger NPs are formed because of the increasing mixing efficiency. At large Weber numbers, there is a transition into transmitive atomization. At this point, an unstable rim is seen with droplets leaving the main body of fluid, resulting in a net decrease in mixing efficiency. Within these droplets, it is possible that larger NPs are formed especially if they are silver nitrate rich.

The reduction in size and dispersity is more obvious for the $0.5 \mathrm{~mm}$ IJR. This reduction is obtained as the mixing efficiency is increased within the liquid sheet, causing the average size of the NPs to decrease and minimizing the formation of larger NPs. Again, this is because the reactions are taking place with a more efficient spread of sodium borohydride, which increases stability of formed NPs and decreases the formation of NPs in silver nitrate rich regions. There is reduction in size and polydispersity when the hydrodynamics of the IJR transition from a uniform liquid sheet (We <29) to a liquid sheet with waves (We $>39$ ) with the size and polydispersity ultimately reducing from $7.9 \pm 5.8 \mathrm{~nm}$ to $3.4 \pm 1.4 \mathrm{~nm}$.

3.2.2. Effect of Weber Number on Silver NP Size and Dispersity Using PVA as a Stabilizing Agent. The Weber number effect on silver NPs was also investigated in the $0.5 \mathrm{~mm}$ and $0.25 \mathrm{~mm}$ IJRs using PVA as a stabilizing agent. Concentration of input 1 was $0.9 \mathrm{mM}$ silver nitrate and 0.02 wt.\% PVA, and 

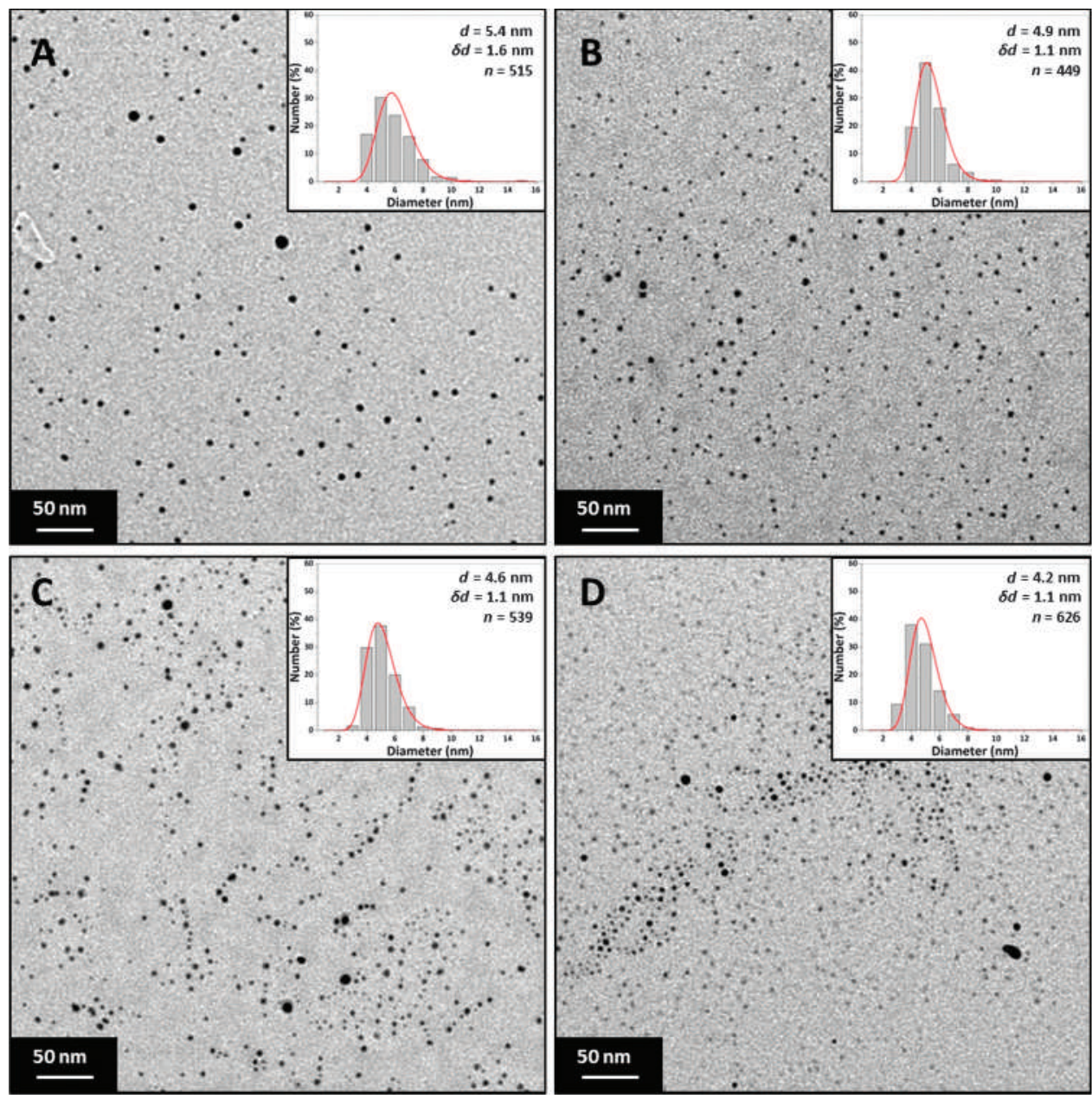

Figure 8. TEM images and particle size distributions of silver NPs synthesized at different total flow rates using a 0.5 -mm I.D. tubing IJR. (A) $32 \mathrm{~mL} / \mathrm{min}$ (We: 13), (B) $48 \mathrm{~mL} / \mathrm{min}$ (We: 29), (C) $64 \mathrm{~mL} / \mathrm{min}$ (We: 51), and (D) $72 \mathrm{~mL} / \mathrm{min}$ (We: 65). Concentration of silver nitrate, $0.9 \mathrm{mM} ;$ PVA, $0.02 \mathrm{wt} . \%$ in input 1 ; and sodium borohydride, $1.8 \mathrm{mM}$ in input 2

concentration of input 2 was $1.8 \mathrm{mM}$ sodium borohydride. Total flow rates for the $0.5 \mathrm{~mm}$ IJR ranged from $32 \mathrm{~mL} / \mathrm{min}$ to $72 \mathrm{~mL} / \mathrm{min}$ and, for the $0.25 \mathrm{~mm} \mathrm{IJR}$, ranged from $18 \mathrm{~mL} / \mathrm{min}$ to $42 \mathrm{~mL} / \mathrm{min}$ (repeatability of the synthesis is shown in Supporting Information, Figure S5). PVA as a surfactant affects the surface tension of the aqueous solution. The surface tension for water is $72 \mathrm{mN} / \mathrm{m}$, and it is estimated that PVA reduces it to a value between 60 and $65 \mathrm{mN} / \mathrm{m}$ [49]. Although there is a small increase in Weber number because of reduced surface tension using PVA, the hydrodynamics observed showed minimal change when compared to using water only (see Supporting Information, Figure S6).

Figure 8 shows TEM images for NPs synthesized in the $0.5 \mathrm{~mm}$ IJR at 32, 48, 64, and $72 \mathrm{~mL} / \mathrm{min}$, where the average diameter and dispersity of the NPs are $5.4 \pm 1.6 \mathrm{~nm}, 4.9 \pm 1.1 \mathrm{~nm}, 4.6 \pm 1.1 \mathrm{~nm}$, and $4.2 \pm 1.1 \mathrm{~nm}$. Using this jet diameter, the NP size decreases with increasing Weber number (up to a maximum of We: 65). $t$-Tests yielded $p$-values $<0.001$ when comparing the PSDs. The dispersity of the NPs does not change significantly with increasing Weber number. One of the differences from the trisodium citrate stabilized system is a notable lack of larger NPs, resulting in lower polydispersity. This difference can be attributed to the PVA molecules used as ligands which provide a bulkier steric stabilization as opposed to the electrostatic stabilization of the smaller citrate molecule, leading to a stronger barrier to prevent growth through coalescence of NPs.

Figure 9 shows TEM images for NPs synthesized in the $0.25 \mathrm{~mm} \mathrm{IJR}$ at $18,26,34$, and $42 \mathrm{~mL} / \mathrm{min}$, where the average diameter and dispersity of the NPs are $4.3 \pm 1.0 \mathrm{~nm}, 4.7 \pm$ $1.3 \mathrm{~nm}, 4.3 \pm 0.7 \mathrm{~nm}$, and $4.6 \pm 1.1 \mathrm{~nm}$. The size and dispersity do not seem to be correlated with the Weber number in this case. $t$-Tests yielded $p$-values of 0.68 when comparing 18 and $34 \mathrm{~mL} / \mathrm{min}, 0.22$ when comparing 26 and $42 \mathrm{~mL} / \mathrm{min}$, and $<0.001$ in all other cases. It appears that, in the $0.25 \mathrm{~mm} \mathrm{IJR}$, there is not a discernible change of NP size when compared to the $0.5 \mathrm{~mm}$ jets, especially with $p$-values suggesting there is no difference between 18 and $34 \mathrm{~mL} / \mathrm{min}$ and 26 and $42 \mathrm{~mL} / \mathrm{min}$.

Figure 10 shows the peak absorbance vs. Weber number obtained from UV-vis spectroscopy for the silver NPs obtained using the $0.5 \mathrm{~mm} \mathrm{IJR}(\mathrm{We}=13-65)$ and the $0.25 \mathrm{~mm} \mathrm{IJR}$ (We = 32-176). In terms of stability, the UV signal changed in the first 15 min after synthesis but remained stable after 30 min and up to $2 \mathrm{~h}$. After $24 \mathrm{~h}$, there was a $16 \%$ increase in peak absorbance. For the $0.5 \mathrm{~mm}$ IJR, the peak absorbance decreases with increasing Weber number. For the $0.25 \mathrm{~mm} I J R$, the peak absorbance decreases minimally as the Weber number increases. This indicates that, in the $0.5 \mathrm{~mm}$ IJR case, the NP size decreases with increasing Weber number, which is in agreement with the TEM observations. In the $0.25 \mathrm{~mm}$ IJR case, the peak absorbance trend indicates that the NP size changes minimally with increasing Weber number, again, in agreement with TEM observations.

PVA has a much higher molecular weight than trisodium citrate and is expected to be more effective at stabilizing the NPs through adsorption onto the silver NP surface through its hydroxyl groups, which are numerous along the chain [50]. Citrate molecules, which are much smaller, adsorb on the surface of the NPs through their carboxyl groups (citrate has three carboxyl groups as opposed to the many hydroxyl groups on a 

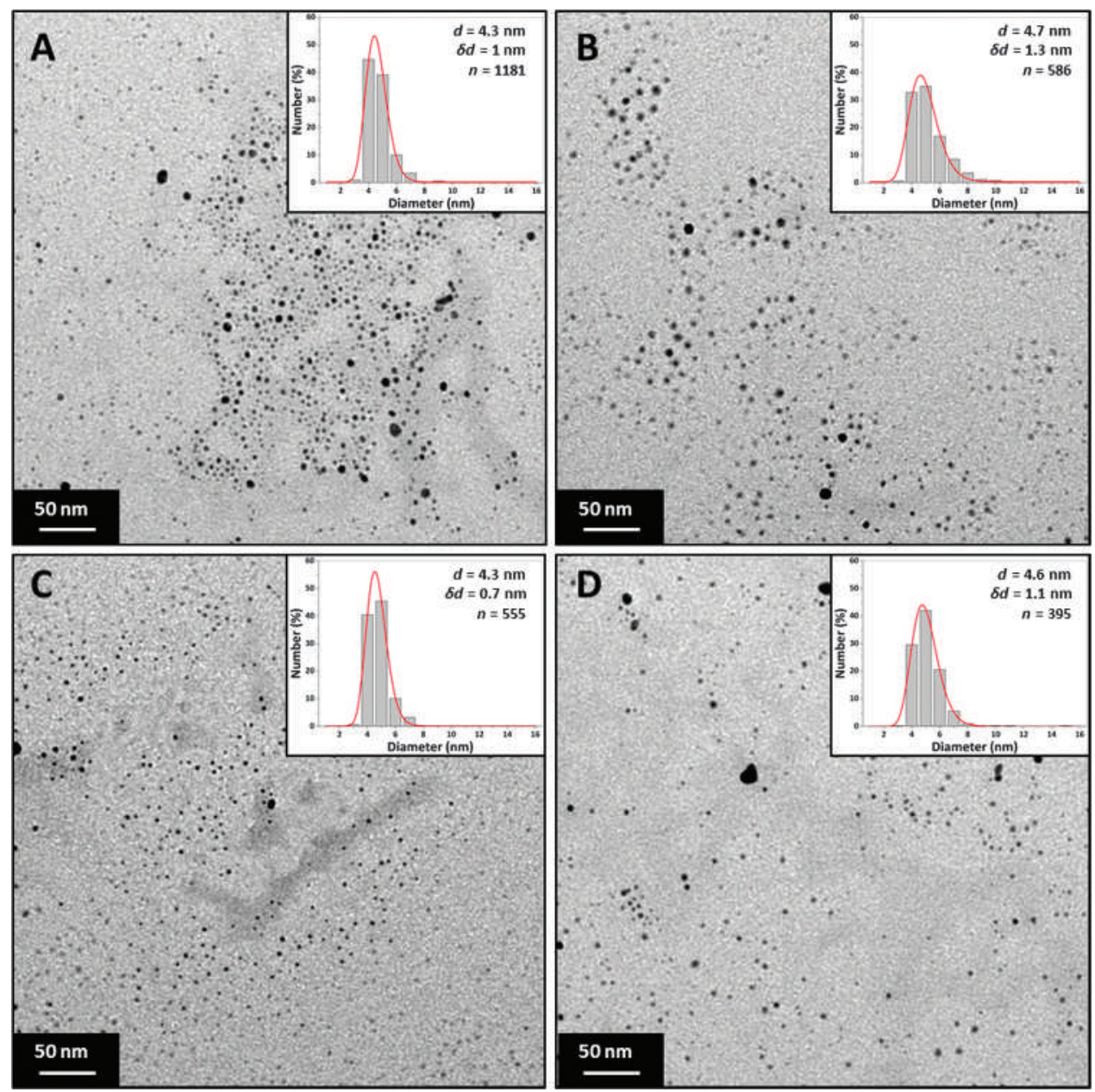

Figure 9. TEM images and particle size distributions of silver NPs synthesized at different total flow rates using a 0.25 -mm I.D. tubing IJR. (A) $18 \mathrm{~mL} / \mathrm{min}$ (We: 32), (B) $26 \mathrm{~mL} / \mathrm{min}$ (We: 68), (C) $34 \mathrm{~mL} / \mathrm{min}$ (We: 115), and (D) $42 \mathrm{~mL} / \mathrm{min}$ (We: 176). Concentration of silver nitrate, $0.9 \mathrm{mM}$; PVA, 0.02 wt. $\%$ in input 1 ; and sodium borohydride, $1.8 \mathrm{mM}$ in input 2

PVA chain) [51]. Another aspect of the two ligands is the effect they have on the kinetics of the reduction reaction and subsequent nucleation of NPs. It is possible that the ligands interact with the precursor in different ways; trisodium citrate behaves

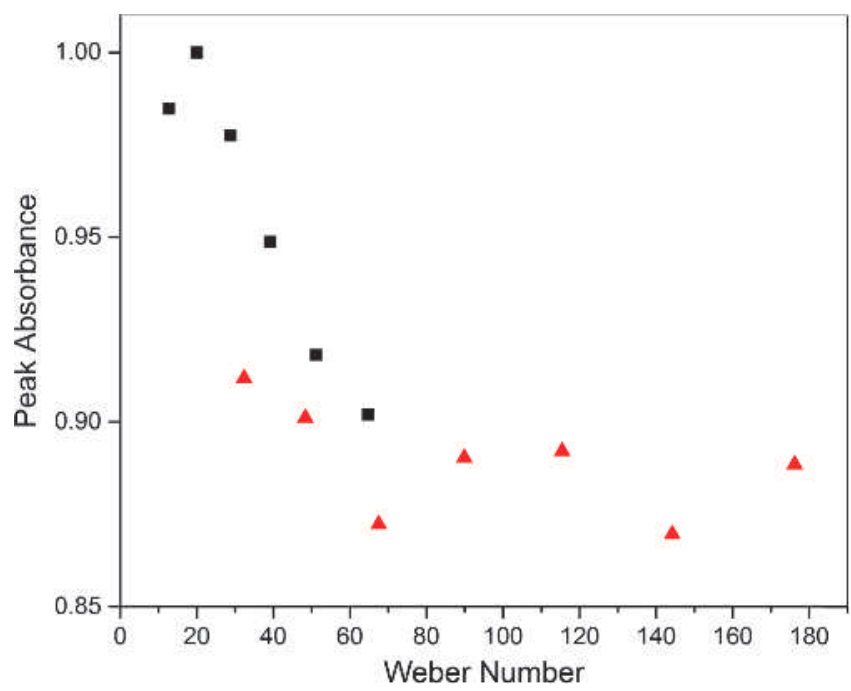

Figure 10. Dependence of peak absorbance (peak wavelength 398$401 \mathrm{~nm}$ ) on Weber number obtained from UV-vis spectroscopy of silver NPs synthesized at different total flow rates using: (1) $0.5 \mathrm{~mm}$ (black squares) and (2) $0.25 \mathrm{~mm}$ (red triangles) I.D. tubing IJR. Concentration of silver nitrate, $0.9 \mathrm{mM}$; PVA, $0.02 \mathrm{wt} . \%$ in input 1 ; and sodium borohydride, $1.8 \mathrm{mM}$ in input 2 as a buffer and affects the solution $\mathrm{pH}$, whereas PVA does not behave in this manner. Literature indicates that the PVA molecule slows down growth of silver NPs compared to reduction with no ligand present, by investigating the evolution of absorbance over time [52]. This reduction in growth rate is most likely because of the bulky nature of the PVA, which would make it more difficult for clusters of silver to approach a growing nucleus. It is suggested that, since trisodium citrate molecules are smaller than PVA, they would allow a closer approach for silver clusters in solution to the NP surface (literature suggests various cluster sizes such as formation of $\mathrm{Ag}_{13}$ and $\mathrm{Ag}_{2}{ }^{+}$) [53]. The presence of highly concentrated silver nitrate zones in the liquid using an IJR in the case of citrate capped NPs will likely result in growth at the surface of the NP through coalescence of silver clusters. The possible reason that no larger NPs are seen using the PVA is that it provides a much larger barrier on the NP surface, inhibiting the approach of silver clusters and rapid growth in areas that are rich in silver nitrate. At the same time, the stabilizing effect of borohydride can be taken into account. There is a balance between how quickly reduced silver clusters can approach and incorporate onto the NP surface vs. the abundance of borohydride in the vicinity of the NP. As mixing efficiency increases, there is a better distribution of borohydride, resulting in a suppressed growth rate and smaller NPs, which is the observed effect using PVA as a ligand in the $0.5 \mathrm{~mm}$ IJR. Using the $0.25 \mathrm{~mm}$ IJR, which yields a more constant size with increasing flow rate, the size of the NPs does not fall below ca. $4 \mathrm{~nm}$ with increasing mixing efficiency. The size of the NPs using the $0.25 \mathrm{~mm}$ IJR fluctuates between 4.3 
and $4.7 \mathrm{~nm}$. The reason for this fluctuation seems to be due to the flow regime in the $0.25 \mathrm{~mm}$ IJR, with the unstable rim region contributing to a fluctuating size which is not as repeatable as a stable rim. The repeatability data for the PVA system shown in Supporting Information (Figure S5) also indicates that silver NPs synthesized in the $0.25 \mathrm{~mm}$ IJR show more fluctuation in absorbance as compared to the $0.5 \mathrm{~mm}$ IJR, further suggesting that the unstable rim contributes to the fluctuating NP size in the $0.25 \mathrm{~mm}$ IJR system. It is expected that improved mixing efficiency should result in a reduction in size as explained above and observed in the $0.5 \mathrm{~mm}$ IJR. However, this reduction in size is not seen with improving mixing efficiency in the $0.25 \mathrm{~mm}$ IJR because the PVA may be imposing a limit on the size of NPs. The synthesis was repeated in the $0.5 \mathrm{~mm}$ IJR at a $\mathrm{We}=65$ using double the concentration of PVA (0.04 wt.\%). The size was reduced from $4.2 \pm 1.1 \mathrm{~nm}$ to $3.7 \pm$ $1.0 \mathrm{~nm}$, suggesting that the PVA concentration is one of the factors that determine the NP size (TEM image and PSD for this experiment are shown in Supporting Information, Figure S7). This suggests that, to achieve NPs smaller than $4 \mathrm{~nm}$, an increase in PVA would be required; otherwise, once a certain mixing efficiency is achieved, the size of the NP does not decrease at a fixed PVA concentration.

\section{Conclusions}

The impinging jet reactor (IJR) was characterized visually and using the Villermaux-Dushman test reaction system to investigate the flow regime and mixing efficiency under a range of flow rates and jet diameters of $0.25 \mathrm{~mm}$ and $0.5 \mathrm{~mm}$. A maximum mixing efficiency was identified at a Weber number of ca. 90, and it was found to correspond to the point at which the flow regime transitions from a stable rim sheet/chain-like pattern to an unstable rim according to visual observation of the impinging jets. This point is identified as the transition from a reflective to a transmitive type atomization.

Two different ligands for silver NP synthesis were investigated using the IJR: trisodium citrate, which electrostatically stabilizes NPs, and PVA, which is a large chain molecule that sterically stabilizes NPs. Trisodium citrate produced more polydisperse silver NPs. Average size was reduced with increasing flow rate because of increased mixing efficiency, reducing the silver nitrate rich zones occurring in the mixing zone after impingement. Increasing mixing efficiency in the PVA system past a Weber number ca. 60 did not yield smaller size NPs, suggesting a limit of size achievable using PVA as a ligand, possibly due to the small amount of PVA present. More monodispersed NPs are obtained using PVA, indicating that it is more efficient at stabilizing the NPs since it has more potential sites available for adsorption onto the surface of the NP than the citrate molecule, and it kinetically hinders growth during NP formation. The lack of fouling and the efficient mixing in the IJR are the main benefits for the synthesis of NPs. The size of the NPs can be reduced with increasing mixing efficiency while also reducing polydispersity. The IJR is scalable through a "scale-out" procedure of essentially increasing the number of IJRs operating in tandem, making it suitable for large scale synthesis of NPs.

Acknowledgments. The authors would like to thank EPSRC and University College London for funding. N. T. K. Thanh thanks The Royal Society for her University Research Fellowship.

Open Access. This article is distributed under the terms of the Creative Commons Attribution 4.0 International License (https://creativecommons.org/licenses/by/4.0/), which permits unrestricted use, distribution, and reproduction in any medium, provided the original author and source are credited, you give a link to the Creative Commons License, and indicate if changes were made.

\section{Supporting Information}

Electronic Supplementary Material (ESM) can be found in the online version at doi: 10.1556/1846.2016.00015.

\section{References}

1. Roduner, E. Chem. Soc. Rev. 2006, 35, 583-592

2. Marambio-Jones, C.; Hoek, E. M. J. Nanopart. Res. 2010, 12, 1531-1551. 1735.

3. Jiang, Z.-J.; Liu, C.-Y.; Sun, L.-W. J. Phys. Chem. B 2005, 109, 1730

4. Yang, Y.; Matsubara, S.; Xiong, L.; Hayakawa, T.; Nogami, M. J. Phys. Chem. C 2007, 111, 9095-9104.

5. Chen, D.; Qiao, X.; Qiu, X.; Chen, J. J. Mater. Sci. 2009, 44, 1076-1081. 6. McFarland, A. D.; Van Duyne, R. P. Nano Lett. 2003, 3, 1057-1062.

7. Thanh, N. T. K.; Maclean, N.; Mahiddine, S. Chem. Rev. 2014, 114, 7610-7630.

8. Agnihotri, S.; Mukherji, S.; Mukherji, S. RSC Adv. 2014, 4, 3974-3983.

9. Sun, Y.; Xia, Y. Adv. Mater. 2003, 15, 695-699.

10. Maillard, M.; Giorgio, S.; Pileni, M.-P. Adv. Mater. 2002, 14, 1084. 416.

11. Busbee, B. D.; Obare, S. O.; Murphy, C. J. Adv. Mater. 2003, 15, 414

12. Hugounenq, P.; Levy, M.; Alloyeau, D.; Lartigue, L.; Dubois, E.; Cabuil, V.; Ricolleau, C.; Roux, S.; Wilhelm, C.; Gazeau, F.; Bazzi, R. J. Phys. Chem. C 2012, 116, 15702-15712.

13. Bakr, O. M.; Wunsch, B. H.; Stellacci, F. Chem. Mater. 2006, 18, $3297-$ 3301 .

14. Khurshid, H.; Chandra, S.; Li, W.; Phan, M. H.; Hadjipanayis, G. C.; Mukherjee, P.; Srikanth, H. J. Appl. Phys. 2013, 113, 17B508.

15. Murphy, C. J.; Jana, N. R. Adv. Mater. 2002, 14, 80.

16. Shevchenko, E. V.; Bodnarchuk, M. I.; Kovalenko, M. V.; Talapin, D. V.; Smith, R. K.; Aloni, S.; Heiss, W.; Alivisatos, A. P. Adv. Mater. 2008, 20, 4323 4329

17. Shen, X.; Song, Y.; Li, S.; Li, R.; Ji, S.; Li, Q.; Duan, H.; Xu, R.; Yang, W.; Zhao, K. RSC Adv. 2014, 4, 34179-34188.

18. Gavriilidis, A.; Angeli, P.; Cao, E.; Yeong, K. K.; Wan, Y. S. S. Chem. Eng. Res. Design 2002, 80, 3-30.

19. (a) Marre, S.; Jensen, K. F. Chem. Soc. Rev. 2010, 39, 1183-1202; (b) Zhao, C.-X.; He, L.; Qiao, S. Z.; Middelberg, A. P. J., Nanoparticle synthesis in microreactors. Chem. Eng. Sci. 2011, 66, 1463-1479.

20. Baber, R.; Mazzei, L.; Thanh, N. T. K.; Gavriilidis, A. RSC Adv. 2015, 5 , 95585-95591.

21. Wagner, J.; Tshikhudo, T. R.; Koehler, J. M. Chem. Eng. J. 2008, 135, S104-S109.

22. Shirtcliffe, N.; Nickel, U.; Schneider, S. J. Colloid Interface Sci. 1999, $211,122-129$

23. (a) Pinto, V. V.; Ferreira, M. J.; Silva, R.; Santos, H. A.; Silva, F.; Pereira, C. M. Colloids Surf., A 2010, 364, 19-25; (b) Song, K.; Lee, S.; Park, T.; Lee, B. Korean J. Chem. Eng. 2009, 26, 153-155; (c) Creighton, J. A.; Blatchford, C. G.; Albrecht, M. G. J. Chem. Soc., Faraday Trans. 1979, 75, 790-798.

24. Knauer, A.; Csáki, A.; Möller, F.; Hühn, C.; Fritzsche, W.; Köhler, J. M. J. Phys. Chem. C 2012, 116, 9251-9258.

25. Takagi, M.; Maki, T.; Miyahara, M.; Mae, K. Chem. Eng. J. 2004, 101, 269-276.

26. Wagner, J.; Kohler, J. M. Nano Lett. 2005, 5, 685-691.

27. Sohn, C. H.; Seol, W. S.; Shibanov, A. A.; Pikalov, V. P. J. Propul. Power 2007, 23, 131-139.

28. Mahajan, A. J.; Kirwan, D. J. AIChE J. 1996, 42, 1801-1814

29. Mullin, J. W. Crystallization. Butterworth-Heinemann: Oxford, 2001.

30. Erni, P.; Elabbadi, A. Langmuir 2013, 29, 7812-7824.

31. Kumar, D. R.; Prasad, B. L.; Kulkarni, A. A. Ind. Eng. Chem. Res. 2013 $52,17376-17382$.

32. Hosni, M.; Farhat, S.; Hinkov, I.; Ben Amar, M.; Kanaev, A.; Jouini, N. AIChE J. 2015.

33. Johnson, B. K.; Prud'homme, R. K. Aust. J. Chem. 2003, 56, 1021-1024.

34. Johnson, B. K.; Prud'homme, R. K. Phys. Rev. Lett. 2003, 91, 118302.

35. Marchisio, D. L.; Rivautella, L.; Barresi, A. A. AIChE J. 2006, 52, $1877-$ 1887

36. Schwarzer, H. C.; Peukert, W. Chem. Eng. Technol. 2002, 25, 657-661.

37. Siddiqui, S. W.; Zhao, Y.; Kukukova, A.; Kresta, S. M. Ind. Eng. Chem. Res. 2009, 48, 7945-7958.

38. Guichardon, P.; Falk, L. Chem. Eng. Sci. 2000, 55, 4233-4243.

39. Commenge, J.-M.; Falk, L. Chem. Eng. Process. Process Intensif. 2011, 50, 979-990.

40. (a) Falk, L.; Commenge, J. M. Chem. Eng. Sci. 2010, 65, 405-411; (b) Villermaux, J.; Falk, L. Chem. Eng. Sci. 1994, 49, 5127-5140.

41. Bergeron, V.; Bonn, D.; Martin, J. Y.; Vovelle, L. Nature 2000, 405, 772 775.

42. Kashid, M.; Renken, A.; Kiwi-Minsker, L. Chem. Eng. J. 2011, 167, 436443.

43. (a) Bush, J. W.; Hasha, A. E. J. Fluid Mech. 2004, 511, 285-310; (b) Bremond, N.; Villermaux, E. J. Fluid Mech. 2006, 549, 273-306.

749 
45. Paramelle, D.; Sadovoy, A.; Gorelik, S.; Free, P.; Hobley, J.; Fernig, D. G. Analyst 2014, 139, 4855-4861.

46. Van Hyning, D. L.; Zukoski, C. F. Langmuir 1998, 14, 7034-7046.

47. Van Hyning, D. L.; Klemperer, W. G.; Zukoski, C. F. Langmuir 2001, 17, $3120-3127$

48. (a) Haynes, W. M. CRC Handbook of Chemistry and Physics, 95th ed.; CRC Press, Taylor \& Francis: Boca Raton, Fla. [u. a.]; London, 2014; (b) Fanning, J. C.; Brooks, B. C.; Hoeglund, A. B.; Pelletier, D. A.; Wadford, J. A. Inorg. Chim. Acta 2000, 310, 115-119; (c) Shen, J.; Li, Z.; Yan, Q.; Chen, Y. J. Phys. Chem. 1993, 97, 8504-8511.
49. Bhattacharya, A.; Ray, P. J. Appl. Polym. Sci. 2004, 93, 122-130. 50. (a) Chou, H. L.; Wu, C. M.; Lin, F. D.; Rick, J. AIP Adv, 2014, 4, 087111 (b) Khanna, P. K.; Gokhale, R.; Subbarao, V. V. V. S.; Vishwanath, A. K.; Das, B. K.; Satyanarayana, C. V. V. Mater. Chem. Phys. 2005, 92, 229-233.

51. Mpourmpakis, G.; Vlachos, D. G. Phys. Rev. Lett. 2009, 102, 155505. 52. Patakfalvi, R.; Virányi, Z.; Dékány, I. Colloid Polym. Sci. 2004, 283 299-305.

53. (a) Takesue, M.; Tomura, T.; Yamada, M.; Hata, K.; Kuwamoto, S.; Yonezawa, T. J. Am. Chem. Soc. 2011, 133, 14164-14167; (b) Pillai, Z. S.; Kamat, P. V. J. Phys. Chem. B 2003, 108, 945-951. 